\title{
Risk Factors for Endophthalmitis Following Open Globe Injuries: A 17-Year Analysis
}

\author{
Asad F Durrani (D) \\ Peter Y Zhao' \\ Yunshu Zhou (iD) \\ Michael Huvard ${ }^{2}$ \\ Lyna Azzouz ${ }^{3}$ \\ Jason M Keil $\mathbb{D}^{3}$ \\ Stephen T Armenti ${ }^{1}$ \\ Vaidehi S Dedania ${ }^{4}$ \\ David C Musch ${ }^{1,5}$ \\ David N Zacks' \\ 'Department of Ophthalmology and \\ Visual Sciences, W.K. Kellogg Eye Center, \\ University of Michigan, Ann Arbor, MI, \\ USA; ${ }^{2}$ Department of Ophthalmology, \\ University of Colorado School of \\ Medicine, Aurora, CO, USA; ${ }^{3}$ University \\ of Michigan Medical School, Ann Arbor, \\ MI, USA; ${ }^{4}$ Department of \\ Ophthalmology, NYU Grossman School \\ of Medicine, New York, NY, USA; \\ ${ }^{5}$ Department of Epidemiology, School of \\ Public Health, University of Michigan, \\ Ann Arbor, MI, USA
}

Background/Aims: To determine the rate of endophthalmitis and assess risk factors for development of endophthalmitis following open globe injury (OGI).

Methods: A retrospective chart review of all patients treated for OGI at the University of Michigan from January 2000 to July 2017 was conducted. Exclusion criteria included intravitreal injection or intraocular surgery in the 30 days prior to injury or less than 30 days of follow-up. A total of 586 out of 993 open globe injuries were included in the study. The main outcome measure was the rate of endophthalmitis.

Results: In this study, 25/586 eyes (4.3\%) had endophthalmitis. Of these, 12/25 eyes (48.0\%) presented with endophthalmitis and 13/25 eyes $(52.0 \%)$ developed endophthalmitis after globe closure. Multivariate analysis identified time to globe repair (OR 4.5, CI 1.9-10.7, $\mathrm{p}=0.0008$ ), zone I injury (OR 3.6, CI 1.1-11.0, $\mathrm{p}=0.0282$ ), and need for additional surgery (OR 5.5, CI 1.5-19.7, $\mathrm{p}=0.0092$ ) as factors associated with increased risk of developing endophthalmitis. Subconjunctival antibiotic injection at the time of globe closure (OR 0.3, CI $0.1-0.7, p=0.0036$ ) was associated with decreased risk of developing endophthalmitis.

Conclusion: Prompt globe closure and subconjunctival antibiotics may reduce the risk of endophthalmitis in OGI. Furthermore, our practice of a one-time dose of systemic prophylactic antibiotics, and intravitreal antibiotics if intraocular foreign body (IOFB) removal is delayed, was not found to increase the rate of endophthalmitis.

Keywords: endophthalmitis, intraocular foreign body, ocular trauma, open globe injury

\section{Introduction}

Open globe injuries (OGI) are often visually devastating and frequently managed at tertiary referral centers around the world. The incidence of OGI in the United States is 4.49 per 100,000 persons with an estimated cost of $\$ 793$ million to the healthcare system between 2006 and 2014. ${ }^{1}$ Visual acuity (VA) is often poor following OGI due to a variety of factors, with endophthalmitis being one of the most devastating complications. The reported rate of endophthalmitis following OGI varies from $0.9 \%$ to $17 \%$. $^{2-11}$ The most commonly isolated organisms from cases of posttraumatic endophthalmitis include Streptococcus species (16.9-21.8\%), ${ }^{12,13}$ Staphylococcus species $\quad(12.0-15.6 \%),{ }^{12,13}$ and Bacillus species (8.7-50.0\%). 6,7,9,14 Bacillus species are well known for causing fulminant endophthalmitis following injury involving soil. Previous investigations have found the presence of an intraocular foreign body (IOFB), ${ }^{2,3,5,6,15}$ delay in time to closure, $5,6,13,15$ lens capsule violation, ${ }^{5,6}$ primary intraocular lens placement at time of globe closure, ${ }^{2}$ isolated corneal injury, ${ }^{5}$ and lacerating mechanism of injury, ${ }^{8}$ as risk factors for the development of endophthalmitis following OGI.
Department of Ophthalmology and Visual Sciences, W.K. Kellogg Eye Center, University of Michigan, 1000 Wall Street, Ann Arbor, MI, 48105, USA

Tel +l 734-232-8404

Email Davzacks@med.umich.edu 
No consensus exists regarding management practices to best prevent endophthalmitis in OGI. While the literature has consistently shown that expedient globe closure is prudent, the question of when to remove an intraocular foreign body remains unclear. Furthermore, the use of prophylactic intravitreal antibiotics, as well as the route and duration of systemic antibiotics varies among centers. As a tertiary referral center, the University of Michigan manages a large number of OGI. Over time, a shift in the choice of prophylactic antibiotic route and duration occurred with an emphasis on a one-time dose of intravenous (IV) antibiotics administered in the emergency department (ED) prior to prompt globe closure as opposed to routine admission for IV antibiotics. In addition, removal of IOFBs at the time of globe closure is not always feasible, and in these cases intravitreal antibiotics are administered at the time of globe closure. The purpose of this study was to assess the risk factors and protective factors for the development of endophthalmitis following OGI.

\section{Methods}

The Institutional Review Board (IRB) of the University of Michigan approved this study prior to data collection and waved the need for informed consent. This study was compliant with the Health Insurance Portability and Accountability Act (HIPAA) and adhered to the tenets of the Declaration of Helsinki. Following IRB approval, a retrospective review of all OGI managed surgically at the University of Michigan between January 2000 and July 2017 was conducted. Inclusion criteria included diagnosis and surgical management of OGI during the study period with a minimum follow-up time of 30 days. Exclusion criteria included less than 30 days of followup (231), repair of injury at an outside institution (101), inadequate records (69), death prior to repair of injury (3), self-sealing injuries that did not require surgical management (2), iatrogenic open globe injury created during intraocular surgery (1). Prior to data collection, it was planned to exclude patients with prior history of endophthalmitis, history of intravitreal injection of any medication within 30 days prior to injury, and history of intraocular or extraocular surgery for a condition unrelated to OGI within 30 days of injury, however, no such cases were identified. Eyes that underwent enucleation within 30 days of injury unless diagnosed with endophthalmitis prior to enucleation were not included in statistical analysis when determining the risk factors for the development of endophthalmitis.

Clinical data including presenting characteristics, medical and surgical management choices, and post-operative outcomes were extracted from the electronic medical record. Snellen VA information from the chart was converted to $\log$ MAR VA for statistical analysis. Count fingers, hand motion, light perception, and no light perception vision were assigned $\operatorname{logMAR}$ values of 1.85 , $2.3,2.75$, and 3.2 , respectively. ${ }^{16}$ Zone of injury was defined utilizing the definitions from the Ocular Trauma Classification Group. ${ }^{17}$

The protocol for the evaluation and repair of OGI is standardized at the University of Michigan. All OGI are seen through the emergency department (ED). Referring EDs are advised to start intravenous antibiotics if possible and place a shield over the injured eye prior to transferring patients. A limited examination of the injured eye is performed in the ED in order to determine if an OGI is present and a complete dilated exam is performed of the fellow eye. Following examination, the injured eye is covered with a shield. IV antibiotics are started if not already given at the referring center. A computed tomographic (CT) scan with thin cuts through the orbits is obtained. Patients are then made nil per os (NPO), given a tetanus booster if needed, provided pain and anti-emetic medication as needed, and consented for globe closure with the on-call trauma team. Globe closure is completed as soon as possible and within 24 hours unless a delay in presentation to the ED had occurred or in cases of polytrauma where the patient is either unstable for surgical intervention or other life-saving surgeries necessitate delay in globe closure. Standard surgical principles are applied to open globe repairs. If an IOFB is present in the posterior segment, and the decision is made to remove the IOFB secondarily, then intravitreal antibiotics are administered at time of globe closure. After operative management, topical antibiotics, topical corticosteroids, and topical cycloplegia are administered. In some cases, PO antibiotics were prescribed based on physician preference. Patients were not routinely admitted for IV antibiotics following OGI. Close follow-up was arranged with sub-specialty care as needed based on the residual pathology post-repair.

Cases of suspected endophthalmitis were identified as those with clinical signs of endophthalmitis including increased pain, decreased vision, increased redness, increased intraocular inflammation, or presence of 
hypopyon. Culture-positive cases were those where an isolate grew and culture-negative cases were those where the clinical definition of endophthalmitis was met with negative culture results.

The primary outcome in this study was the rate of endophthalmitis over the study period. All potential risk factors for the development of endophthalmitis were assessed for their statistical significance in univariate logistic regression models. Next, all significant univariate predictors were evaluated in a multivariable logistic regression model and a final multivariable model was determined using backward selection. A p-value of $<0.05$ was considered statistically significant. All statistical tests were two-sided. Analyses were performed using SAS version 9.4 (SAS Institute, Cary, NC, USA).

\section{Results}

There were 993 OGI diagnosed at the University of Michigan over the study period, of which a total of 586 met inclusion criteria (Table 1). The majority of injuries, $442 / 586$ injuries (75.4\%), occurred in male patients. Mean age of patients was $40.7 \pm 25.1$ years, ranging from 1 to 99 years old. Mean follow-up time was $1079.8 \pm 1895.7$ days with a median follow-up time of 443 days. The majority of eyes, 441/586 (75.3\%), presenting with an OGI had no previous history of ocular surgery. Information on time from injury to presentation was available for 583 of the 586 cases (99.5\%); 77/583 (13.2\%) presented more than 24 hours following injury for a variety of reasons including lack of awareness of the severity of their injury and initially presenting to centers not equipped for surgical management of open globe. Globe closure occurred within 24 hours of injury in $480 / 582(82.5 \%)$ of cases, with indeterminate timing in 4 cases.

OGI often resulted in profound vision loss. Mean presenting logMAR VA was $2.1 \pm 1.0$ (Snellen equivalent between counting fingers and hand motion VA). Mean logMAR VA was $1.3 \pm 1.2$ (Snellen equivalent of 20/400) at the time of last documented follow-up, which was a statistically significant improvement compared to presentation $(\mathrm{p}<0.0001)$. Ultimately, $117 / 586$ eyes $(20.0 \%)$ required enucleation.

A broad array of injury mechanisms and zones of injury were treated at our center. Details of the OGI as well as additional procedures performed at the time of globe closure can be found in Table 2. An IOFB was present in 78 of the 586 cases (14.8\%). Thirty of these IOFBs were located in the anterior chamber and 48 were located posteriorly. For IOFBs removed after primary globe closure, the median time to removal was two days after injury with a range of zero to 28 days. A large number of patients required additional surgery with the median number of additional surgeries being one.

In this study, 25/586 (4.3\%) eyes were suspected to have endophthalmitis (Table 3), with 12 (48.0\%) detected on presentation. Delayed presentation of over 24 hours after injury occurred in 10/25 (40.0\%) of endophthalmitis cases, and $6 / 25(24.0 \%)$ involved an IOFB. Of the 13/25 cases (52.0\%) that developed endophthalmitis following globe closure, the median time to diagnosis of endophthalmitis was five days following presentation. The majority, 14/25 (56.0\%), of patients with endophthalmitis had a Zone 1 injury only. Initial management included tap and injection

Table I Demographics and Summary Features of Open Globe Injuries

\begin{tabular}{|l|c|c|c|}
\hline & Total Number & With Endophthalmitis & Without Endophthalmitis \\
\hline Open Globe Injuries & 586 & 25 & $56 \mathrm{I}$ \\
Male & $442 / 586(75.4 \%)$ & $20 / 25$ & $422 / 56 \mathrm{I}$ \\
Female & $144 / 586(24.6 \%)$ & $5 / 25$ & $139 / 56 \mathrm{I}$ \\
Average age & $40.7 \pm 25.1$ years & $42.0 \pm 29.3$ years & $40.7 \pm 24.9$ years \\
Median follow-up & 443 days (IQR I293) & $792(\mathrm{QQR} I 190.75)$ & 430 (IQR I293) \\
Total cases of suspected endophthalmitis & $25 / 586(4.3 \%)$ & $25 / 25$ & $0 / 56 \mathrm{I}$ \\
Cases of endophthalmitis on presentation & $12 / 586(2.0 \%)$ & $12 / 25$ & $0 / 56 \mathrm{I}$ \\
Cases of endophthalmitis post-globe closure & $13 / 586(2.2 \%)$ & $13 / 25$ & $0 / 56 \mathrm{I}$ \\
Presenting logMAR visual acuity & $2.1 \pm 1.0$ & $2.2 \pm 0.9$ & $2.10 \pm 1.0$ \\
Presenting Snellen equivalent visual acuity & Between count fingers and & Between count fingers and & Between count fingers and \\
& hand motion & hand motion & hand motion \\
LogMAR visual acuity at last follow-up & $1.3 \pm 1.2$ & $1.9 \pm 1.3$ & $1.3 \pm 1.2$ \\
Snellen equivalent visual acuity at last follow- & $20 / 400$ & Between count fingers and & $20 / 400$ \\
up & & hand motion & \\
\hline
\end{tabular}


Table 2 Details of Open Globe Injury and Additional Procedures Performed at Time of Globe Closure

\begin{tabular}{|c|c|c|c|}
\hline Injury Mechanism & Total Number* & With Endophthalmitis & Without Endophthalmitis \\
\hline Rupture & $358 / 586(61.1 \%)$ & $10 / 25(40.0 \%)$ & $348 / 56 \mid(62.0 \%)$ \\
\hline Perforating & $118 / 586(20.1 \%)$ & $8 / 25(32.0 \%)$ & $|I 0 / 56|(19.6 \%)$ \\
\hline Penetrating & $110 / 586(18.8 \%)$ & $7 / 25(28.0 \%)$ & $103 / 56 \mid(18.4 \%)$ \\
\hline \multicolumn{4}{|l|}{ Zones of injury } \\
\hline Zone I & $268 / 584(45.9 \%)$ & $19 / 25(76.0 \%)$ & $249 / 559(44.5 \%)$ \\
\hline Zone II & $136 / 584(23.3 \%)$ & $2 / 25(8.0 \%)$ & I34/559 (24.0\%) \\
\hline Zone III & $180 / 584(30.8 \%)$ & $4 / 25(16.0 \%)$ & $176 / 559(3 \mid .5 \%)$ \\
\hline \multicolumn{4}{|l|}{ Wound length } \\
\hline Less than $5 \mathrm{~mm}$ & $|45 / 53|(27.3 \%)$ & I I/24 (45.8\%) & I34/507 (26.4\%) \\
\hline Between 5 and $10 \mathrm{~mm}$ & $|35 / 53|(25.4 \%)$ & $8 / 24(33.3 \%)$ & $127 / 507(25.1 \%)$ \\
\hline Between II-15 mm & $97 / 531$ (18.3\%) & $2 / 24(8.3 \%)$ & $95 / 507(18.7 \%)$ \\
\hline Between $16-20 \mathrm{~mm}$ & $5 I / 531(9.6 \%)$ & $2 / 24(8.3 \%)$ & $49 / 507(9.7 \%)$ \\
\hline Greater than $20 \mathrm{~mm}$ & $103 / 53 \mid(19.4 \%)$ & $\mathrm{I} / 24(4.2 \%)$ & 102/507 (20.1\%) \\
\hline Lens disruption & $267 / 575(46.4 \%)$ & $1 \mathrm{l} / 22(50.0 \%)$ & $256 / 553(46.3 \%)$ \\
\hline Retinal detachment & $181 / 586(30.9 \%)$ & $2 / 25(8.0 \%)$ & $|79 / 56|(3 \mid .9 \%)$ \\
\hline Uveal prolapse & $40 \mathrm{I} / 586(68.4 \%)$ & I I/25 (44.0\%) & $390 / 561$ (69.5\%) \\
\hline Primary lensectomy & $50 / 586(8.5 \%)$ & $3 / 25(12.0 \%)$ & $47 / 56 \mid(8.4 \%)$ \\
\hline Vitrectomy at time of globe repair & $127 / 586(21.7 \%)$ & $3 / 25(12.0 \%)$ & $|24 / 56|(22.1 \%)$ \\
\hline Manual excision of prolapsed vitreous strands & $97 / 586(16.6 \%)$ & $0 / 25(0.0 \%)$ & $97 / 56 \mid(17.3 \%)$ \\
\hline Pars plana vitrectomy & $24 / 586(4.1 \%)$ & $3 / 25(12.0 \%)$ & $21 / 561(3.7 \%)$ \\
\hline Anterior vitrectomy & $6 / 586(1.0 \%)$ & $0 / 25(0.0 \%)$ & $6 / 561(1.1 \%)$ \\
\hline
\end{tabular}

Note: *Denominators vary depending on availability of each available variable in the electronic medical record.

of intravitreal antibiotics in 18/25 cases $(72.0 \%)$, pars plana vitrectomy with tap and injection of intravitreal antibiotics in $4 / 25$ cases $(16.0 \%)$, and primary enucleation in $3 / 25$ cases $(12.0 \%)$. Only $12 / 25$ cases $(48.0 \%)$ were culturepositive; organisms cultured included Bacillus cereus (2), coagulase negative Staphylococcus (2), Candida albicans (1), Clostridium bifermentans (1), Enterococcus faecalis (1), Group A Streptococcus (1), Haemophiles influenzae (1), and Streptococcus pneumoniae (1). Two of the enucleated eyes were found to have Gram-positive rods on pathology. Of the 12 culture-positive cases, five had sensitivity and resistance data available and only one coagulasenegative Staphylococcus isolate showed intermediate fluoroquinolone sensitivity. The most frequently utilized combination of intravitreal antibiotics was vancomycin and ceftazidime in 16/25 cases (72.0\%). Final VA was poor with mean $\log$ MAR VA $1.9 \pm 1.3$, a Snellen equivalent between count fingers and hand motion vision. Five eyes required enucleation.

Following univariate analyses, multivariable logistic regression analysis was performed to assess for statistically significant risk factors and protective factors (Table 4). Intravitreal antibiotics were not included in multivariable analysis despite the suggestion of being a statistically significant risk factor in univariate modeling as this was not a true risk factor but a treatment once endophthalmitis developed resulting in this association. Time to globe repair (OR 4.5, CI 1.9-10.7, $\mathrm{p}=0.0008$ ), zone I injury (OR 3.6, CI 1.1-11.0, p = 0.0282), and the need for additional surgery (OR 5.5, CI 1.5-19.7, $\mathrm{p}=$ 0.0092) were identified as risk factors for the development of endophthalmitis (Table 3 ). Given our study period spanned many years, numerous antibiotic regimens were utilized and subsequently analyzed to assess if any were particularly effective in preventing endophthalmitis. Subconjunctival antibiotics (OR 0.3, CI $0.1-0.7, \mathrm{p}=$ $0.0036)$ were found to be associated with decreased risk of endophthalmitis and utilized in 439/586 cases (74.9\%). Three hundredfifteen eyes received a subconjunctival cephalosporin, 93 eyes received subconjunctival vancomycin, 64 eyes received subconjunctival ceftazidime, and 14 eyes received a subconjunctival aminoglycoside. The 
Table 3 Clinical and Microbiological Characteristics of Endophthalmitis Cases Following Open Globe Injury

\begin{tabular}{|c|c|c|c|c|c|c|c|c|}
\hline Patient & Age & $\begin{array}{l}\text { Injury } \\
\text { Mechanism }\end{array}$ & $\begin{array}{l}\text { Time to } \\
\text { Presentation } \\
\text { and Surgery }\end{array}$ & Injury & $\begin{array}{l}\text { Onset of } \\
\text { Endophthalmitis }\end{array}$ & $\begin{array}{l}\text { Treatment of } \\
\text { Endophthalmitis }\end{array}$ & $\begin{array}{l}\text { Culture } \\
\text { Results }\end{array}$ & $\begin{array}{l}\text { Final } \\
\text { Visual } \\
\text { Acuity }\end{array}$ \\
\hline I & 70 & $\begin{array}{l}\text { Dehisced PKP } \\
\text { from fall }\end{array}$ & $<24$ hours & Dehisced PKP & 5 days & Tap and injection & $\begin{array}{l}\text { Candida } \\
\text { albicans }\end{array}$ & HM \\
\hline 2 & 11 & $\begin{array}{l}\text { Hammering } \\
\text { metal }\end{array}$ & $<24$ hours & $\begin{array}{l}\text { Corneal laceration } \\
\text { with IOFB }\end{array}$ & I day & $\begin{array}{l}\text { PPV/PPL/IOFB } \\
\text { removal with tap } \\
\text { and injection }\end{array}$ & $\begin{array}{l}\text { Clostridium } \\
\text { bifermentans }\end{array}$ & Enucleated \\
\hline 3 & 48 & Hammering ice & $>24$ hours & Scleral laceration & On presentation & $\begin{array}{l}\text { PPV/PPL/IOFB } \\
\text { removal with tap } \\
\text { and injection }\end{array}$ & Negative & NLP \\
\hline 4 & 59 & $\begin{array}{l}\text { Plastic shard hit } \\
\text { eye }\end{array}$ & $>24$ hours & Corneal laceration & On presentation & Tap and injection & Negative & $20 / 200$ \\
\hline 5 & 39 & $\begin{array}{l}\text { Metal wire into } \\
\text { eye }\end{array}$ & $<24$ hours & Corneal laceration & I day & $\begin{array}{l}\text { PPV/PPL with tap } \\
\text { and injection }\end{array}$ & Bacillus cereus & HM \\
\hline 6 & 43 & $\begin{array}{l}\text { Metal tool hit } \\
\text { eye }\end{array}$ & $<24$ hours & Corneal laceration & 6 days & Tap and injection & $\begin{array}{l}\text { Coagulase- } \\
\text { negative } \\
\text { Staphylococcus }\end{array}$ & $20 / 30$ \\
\hline 7 & 86 & $\begin{array}{l}\text { Dehisced PKP } \\
\text { from fall }\end{array}$ & $>24$ hours & Dehisced PKP & On presentation & Tap and injection & $\begin{array}{l}\text { Enterococcus } \\
\text { faecalis }\end{array}$ & HM \\
\hline 8 & 6 & $\begin{array}{l}\text { Thrown pen hit } \\
\text { eye }\end{array}$ & $>24$ hours & Corneal laceration & On presentation & Tap and injection & $\begin{array}{l}\text { Group-A } \\
\text { Streptococcus }\end{array}$ & $20 / 50$ \\
\hline 9 & 1 & Cat scratch & $>24$ hours & Scleral laceration & On presentation & Tap and injection & Negative & LP \\
\hline 10 & 9 & $\begin{array}{l}\text { Rupture of } \\
\text { unclear etiology }\end{array}$ & $>24$ hours & Corneal laceration & On presentation & Tap and injection & Negative & $20 / 40$ \\
\hline II & 77 & $\begin{array}{l}\text { Dehisced PKP } \\
\text { from fall }\end{array}$ & $<24$ hours & Dehisced PKP & 3 days & Tap and injection & Negative & $20 / 50$ \\
\hline 12 & 39 & Grinding metal & $<24$ hours & $\begin{array}{l}\text { Corneal laceration } \\
\text { with IOFB }\end{array}$ & On presentation & $\begin{array}{l}\text { PPV/PPL/IOFB } \\
\text { removal with tap } \\
\text { and inject }\end{array}$ & Negative & $20 / 100$ \\
\hline 13 & 3 & $\begin{array}{l}\text { Scissor or fork } \\
\text { into the eye }\end{array}$ & $>24$ hours & Corneal laceration & On presentation & Tap and injection & Negative & $20 / 20$ \\
\hline 14 & 73 & $\begin{array}{l}\text { Hammering } \\
\text { chisel }\end{array}$ & $>24$ hours & Corneal laceration & On presentation & Tap and injection & Negative & $20 / 25$ \\
\hline 15 & 65 & Fall & $<24$ hours & $\begin{array}{l}\text { Corneoscleral } \\
\text { laceration }\end{array}$ & On presentation & Injection alone & No culture & Enucleated \\
\hline 16 & 20 & Wood hit eye & $<24$ hours & $\begin{array}{l}\text { Corneoscleral } \\
\text { laceration }\end{array}$ & 8 days & Tap and injection & Negative & HM \\
\hline 17 & 32 & $\begin{array}{l}\text { Wire into the } \\
\text { eye }\end{array}$ & $<24$ hours & Corneal laceration & 5 days & Tap and injection & $\begin{array}{l}\text { Coagulase- } \\
\text { negative } \\
\text { Staphylococcus }\end{array}$ & NLP \\
\hline 18 & 60 & $\begin{array}{l}\text { High speed } \\
\text { stone hit eye }\end{array}$ & $>24$ hours & $\begin{array}{l}\text { Corneoscleral } \\
\text { laceration }\end{array}$ & On presentation & Tap and injection & Negative & $20 / 800$ \\
\hline
\end{tabular}


Table 3 (Continued).

\begin{tabular}{|c|c|c|c|c|c|c|c|c|}
\hline Patient & Age & $\begin{array}{l}\text { Injury } \\
\text { Mechanism }\end{array}$ & $\begin{array}{l}\text { Time to } \\
\text { Presentation } \\
\text { and Surgery }\end{array}$ & Injury & $\begin{array}{l}\text { Onset of } \\
\text { Endophthalmitis }\end{array}$ & $\begin{array}{l}\text { Treatment of } \\
\text { Endophthalmitis }\end{array}$ & $\begin{array}{l}\text { Culture } \\
\text { Results }\end{array}$ & $\begin{array}{l}\text { Final } \\
\text { Visual } \\
\text { Acuity }\end{array}$ \\
\hline 19 & 91 & $\begin{array}{l}\text { Perforated PKP } \\
\text { due to corneal } \\
\text { ulcer }\end{array}$ & $<24$ hours & Perforated PKP & I day & Tap and injection & $\begin{array}{l}\text { Haemophiles } \\
\text { influenzae }\end{array}$ & NLP \\
\hline 20 & 91 & $\begin{array}{l}\text { Dehisced PKP } \\
\text { from fall }\end{array}$ & $<24$ hours & Dehisced PKP & 20 days & Tap and injection & Negative & LP \\
\hline 21 & 7 & $\begin{array}{l}\text { Metal rod hit } \\
\text { eye }\end{array}$ & $<24$ hours & Corneal laceration & 36 days & Tap and injection & $\begin{array}{l}\text { Streptococcus } \\
\text { pneumonia }\end{array}$ & NLP \\
\hline 22 & 39 & $\begin{array}{l}\text { Grinding non- } \\
\text { metallic } \\
\text { material }\end{array}$ & $<24$ hours & $\begin{array}{l}\text { Corneal laceration } \\
\text { with IOFB }\end{array}$ & 2 days & Primary enucleation & Bacillus cereus & Enucleated \\
\hline 23 & 10 & $\begin{array}{l}\text { Hammering } \\
\text { rocks }\end{array}$ & $>24$ hours & $\begin{array}{l}\text { Corneoscleral } \\
\text { laceration }\end{array}$ & On presentation & Primary enucleation & $\begin{array}{l}\text { Gram-positive } \\
\text { rods }\end{array}$ & Enucleated \\
\hline 24 & 46 & $\begin{array}{l}\text { Barbed wire } \\
\text { into eye }\end{array}$ & $<24$ hours & Corneal laceration & 2 days & Primary enucleation & $\begin{array}{l}\text { Gram-positive } \\
\text { rods }\end{array}$ & Enucleated \\
\hline 25 & 23 & $\begin{array}{l}\text { Hammering } \\
\text { metal }\end{array}$ & $<24$ hours & Corneal laceration & 49 days & Tap and injection & Negative & $20 / 30$ \\
\hline
\end{tabular}

Abbreviations: PKP, penetrating keratoplasty; IOFB, intraocular foreign body; PPV, pars plana vitrectomy; PPL, pars plana lensectomy; HM, hand motion; LP, light perception; NLP, no light perception.

majority of our patients, 407/586 (69.5\%), received IV antibiotics at the time of diagnosis. The most commonly used IV antibiotics included penicillin-based antibiotics, 213/407 cases (52.4\%), fluoroquinolones, 93/407 cases (22.9\%), and a penicillin-based antibiotic combined with a fluoroquinolone, $32 / 407$ cases $(7.9 \%)$. In the vast majority of cases, 368/407 (90.4\%), IV antibiotics were administered as one-time doses. The use of IV antibiotics ( $\mathrm{p}=0.1403)$ or any duration including one-time dosing $(\mathrm{p}=0.0841)$, more than one dose but less than 24 hours total $(\mathrm{p}=0.1588)$, and greater than 24 hours of IV antibiotics ( $\mathrm{p}=0.9792)$ were not found to be statistically significant protective factors against the development of endophthalmitis. Oral antibiotics were utilized in 201/ 586 cases $(34.3 \%)$ for a mean duration of $7.6 \pm 3.0$ days; however, their use was not a protective factor against the development of endophthalmitis $(\mathrm{p}=0.0622)$. Intravitreal antibiotics were utilized in 67/519 cases (12.9\%). Topical antibiotics were utilized post-operatively in 554/586 cases (94.5\%). Furthermore, the presence of an intraocular foreign body was not found to be a statistically significant risk factor for the development of endophthalmitis. At our center, intravitreal antibiotics were used at the time of globe closure if IOFB removal was delayed.

\section{Discussion}

We report an endophthalmitis rate of $4.3 \%$ in eyes with OGI, which is consistent with previously reported rates between $00.9 \%$ and $17.0 \%{ }^{2-11}$ Our protocol for OGI emphasizes a one-time dose of IV antibiotics on presentation to the ED. Given that twelve of the 25 cases of endophthalmitis were diagnosed on presentation, the rate of endophthalmitis developing following globe closure was actually $2.2 \%$, or in $13 / 574$ cases.

Our study identified several risk factors that are associated with development of endophthalmitis. In particular, the presence of zone I injury as a risk factor for the development of endophthalmitis has only been previously reported in another study with smaller sample size of 117 eyes. ${ }^{5}$ The zones of injury are defined as follows: zone 1 involves the cornea and limbus only, zone 2 involves the limbus to 5 millimeters posterior into the sclera, and zone 3 involves the posterior to 5 millimeters from the limbus. ${ }^{17}$ Given that zone I injuries tend to be lacerating in nature, our finding that these injuries increase the risk of 
Table 4 Univariable and Multivariable Models for Risk Factors for the Development of Endophthalmitis Following Open Globe Injury

\begin{tabular}{|c|c|c|}
\hline \multicolumn{3}{|l|}{ Univariate Analysis } \\
\hline Risk Factor & Odds Ratio & P-value \\
\hline IOFB (yes vs no) & 2.934 & 0.0158 \\
\hline $\begin{array}{l}\text { Injury mechanism (penetrating versus } \\
\text { rupture) }\end{array}$ & 2.375 & 0.0871 \\
\hline $\begin{array}{l}\text { Injury mechanism (perforating versus } \\
\text { rupture) }\end{array}$ & 2.516 & 0.0580 \\
\hline $\begin{array}{l}\text { Time to repair ( }>24 \text { hours versus } \\
<24 \text { hours) }\end{array}$ & 4.833 & 0.0002 \\
\hline Zone of injury ( I versus 3) & 3.385 & 0.0291 \\
\hline Zone of injury (2 versus 3 ) & 0.662 & 0.6364 \\
\hline Wound length $(5-10 \mathrm{~mm}$ vs $<5 \mathrm{~mm}$ ) & 0.767 & 0.5819 \\
\hline Wound length (II-I5 mm vs $<5 \mathrm{~mm}$ ) & 0.256 & 0.0812 \\
\hline Wound length ( $16-20 \mathrm{~mm}$ vs $<5 \mathrm{~mm}$ ) & 0.497 & 0.3744 \\
\hline Wound length ( $>20 \mathrm{~mm}$ vs $<5 \mathrm{~mm}$ ) & 0.122 & 0.0455 \\
\hline Uveal prolapse (yes vs no) & 0.338 & 0.0087 \\
\hline IV antibiotics (yes vs no) & 0.544 & 0.1403 \\
\hline $\begin{array}{l}\text { IV antibiotic duration (one-time versus } \\
\text { none) }\end{array}$ & 0.470 & $0.084 I$ \\
\hline $\begin{array}{l}\text { IV antibiotic duration ( }<24 \text { hours but } \\
\text { more than one dose versus none) }\end{array}$ & 2.679 & 0.1588 \\
\hline $\begin{array}{l}\text { IV antibiotic duration ( }>24 \text { hours } \\
\text { versus none) }\end{array}$ & $<0.001$ & 0.9792 \\
\hline Age & 1.002 & 0.7829 \\
\hline Gender & 0.761 & 0.5919 \\
\hline Laterality of injury (OD vs OS) & 1.144 & 0.7431 \\
\hline Previous ocular surgery (yes vs no) & 1.457 & 0.3925 \\
\hline $\begin{array}{l}\text { Time to presentation ( }>24 \text { hours } \\
\text { vs }<24 \text { hours) }\end{array}$ & 4.940 & 0.0002 \\
\hline LogMAR presenting VA & 1.125 & 0.6063 \\
\hline RAPD (yes vs no) & 1.640 & 0.3523 \\
\hline $\begin{array}{l}\text { Foreign body location (vitreous vs } \\
\text { anterior chamber) }\end{array}$ & 4.000 & 0.2106 \\
\hline $\begin{array}{l}\text { Foreign body location (other vs } \\
\text { anterior chamber) }\end{array}$ & 3.500 & 0.3941 \\
\hline Lens status (pseudophakic vs phakic) & 1.278 & 0.6352 \\
\hline Lens status (aphakic vs phakic) & 4.176 & 0.1964 \\
\hline
\end{tabular}

(Continued)
Table 4 (Continued).

\begin{tabular}{|c|c|c|}
\hline \multicolumn{3}{|l|}{ Univariate Analysis } \\
\hline Risk Factor & Odds Ratio & P-value \\
\hline Lens disruption (yes vs no) & 1.157 & 0.7376 \\
\hline Vitreous hemorrhage (yes vs no) & 0.681 & 0.4015 \\
\hline Intraocular inflammation (yes vs no) & 10.931 & $<0.0001$ \\
\hline Retinal detachment (yes vs no) & 0.230 & 0.0478 \\
\hline Days to treatment of RD & 0.072 & 0.6755 \\
\hline $\begin{array}{l}\text { Time to antibiotics ( }>24 \text { hours vs } \\
<24 \text { hours) }\end{array}$ & 5.700 & $<0.0001$ \\
\hline PO antibiotics (yes vs no) & 2.149 & 0.0622 \\
\hline $\mathrm{PO}$ antibiotics duration in days & 1.115 & 0.3436 \\
\hline Intraocular antibiotics (yes vs no) & 18.061 & $<0.0001$ \\
\hline $\begin{array}{l}\text { Subconjunctival antibiotics (yes vs } \\
\text { no) }\end{array}$ & 0.286 & 0.0024 \\
\hline Topical antibiotics (yes vs no) & 0.387 & 0.1417 \\
\hline Subconjunctival steroids (yes vs no) & 0.534 & 0.1290 \\
\hline Topical steroid (yes vs no) & 0.675 & 0.5356 \\
\hline Cycloplegic (yes vs no) & 1.621 & 0.3119 \\
\hline Vitrectomy (Weck-Cel vs none) & $<0.001$ & 0.9524 \\
\hline Vitrectomy (Anterior vs none) & $<0.001$ & 0.9882 \\
\hline Vitrectomy (Posterior vs none) & 2.946 & 0.0998 \\
\hline Primary lensectomy (yes vs no) & 1.544 & 0.4944 \\
\hline Additional surgeries (yes vs no) & 3.786 & 0.0323 \\
\hline Enucleation (yes vs no) & 1.545 & 0.3421 \\
\hline \multicolumn{3}{|l|}{ Multivariable Analysis } \\
\hline Risk factor & Odds Ratio & P-value \\
\hline Delay in Globe Closure & $\begin{array}{l}\mathrm{OR}=4.5(95 \% \\
\mathrm{Cl} \mathrm{I.9-10.7)}\end{array}$ & 0.0008 \\
\hline Zone I vs Zone III Injury & $\begin{array}{l}\mathrm{OR}=3.6(95 \% \\
\mathrm{Cl} I . \mathrm{I}-\mathrm{II} .0)\end{array}$ & 0.0282 \\
\hline Zone II vs Zone III Injury & $\begin{array}{l}\mathrm{OR}=1.0(95 \% \\
\mathrm{Cl} 0.2-5.7)\end{array}$ & 0.9940 \\
\hline Need for Additional Surgery & $\begin{array}{l}\text { OR }=5.5(95 \% \\
\mathrm{Cl} I .5-19.7)\end{array}$ & 0.0092 \\
\hline Subconjunctival Antibiotics & $\begin{array}{l}\mathrm{OR}=0.3(95 \% \\
\mathrm{Cl} 0 . \mathrm{I}-0.7)\end{array}$ & 0.0036 \\
\hline
\end{tabular}

Note: Bolded rows within table denote risk factors and their associated odds ratio and $\mathrm{p}$-value that were statistically significant in univariate modeling. 
endophthalmitis may be explained by the fact that these injuries are more likely to involve direct inoculation of microbes into the eye compared to rupture injuries. Our study also found that time to globe repair greater than 24 hours after injury was a risk factor for the development of endophthalmitis, which is well described in the literature. ${ }^{5,6,13,15}$ We did not find an association between presence of IOFB and an increased the risk of endophthalmitis as previous studies have. However, we hypothesize that our use of prophylactic intravitreal antibiotics in these cases could explain this difference., ${ }^{2,3,5,6,15}$ The need for additional surgery was also predictive of endophthalmitis. This is likely due to a combination of factors such as number of surgeries being a surrogate for the severity of the original injury and that each additional intraocular surgery carries its own risk of endophthalmitis.

Numerous controversies exist in the management of OGI and there is limited consensus on best practices to prevent endophthalmitis. One area of controversy is the role of systemic antibiotic prophylaxis. In our study, no statistically significant protective benefit was observed in those patients who received intravenous antibiotics over those who did not. Similarly, the use of PO antibiotics was not found to have a protective effect in our study. Only the use of subconjunctival antibiotics at the time of globe closure was found to be associated with decreased risk of endophthalmitis following globe closure. It is possible that subconjunctival antibiotics protect against endophthalmitis as they decrease the microbial load present at the site of injury. Though no statistically significant protective effect was found for the use of systemic prophylaxis, the theoretical benefit likely outweighs the risk of a short course of systemic antibiotics. Outstanding questions regarding systemic antibiotics are what combination provides the best prophylaxis against endophthalmitis and what is the optimal duration of treatment.

Systemic vancomycin and ceftazidime are a potent combination that provides coverage of all Gram-positive and Gram-negative organisms, but their penetration into the vitreous under different anatomic states is questionable. $^{18,19}$ At our institution, a shift toward the use of fluoroquinolone antibiotics for endophthalmitis prophylaxis in OGI has occurred over the last decade. Pharmacokinetic studies have demonstrated that systemically administered fluoroquinolones, especially moxifloxacin, a fourth-generation fluoroquinolone, achieve therapeutic levels in the vitreous while covering the most commonly cultured organisms in cases of endophthalmitis. ${ }^{20-22}$ In terms of duration, our study found no protective benefit in those receiving a prolonged course of antibiotics for prophylaxis compared to those receiving a one-time dose. Numerous studies from other surgical fields have found that long courses of prophylactic antibiotics provide no additional protective effect when compared to short courses of antibiotics and may actually increase the frequency of infections with resistant organisms. ${ }^{23-27}$ Given these findings in the literature and our results, we consider a one-time IV dose of a fourth-generation fluoroquinolone as a reasonable choice for prophylaxis in OGI. A one-time dose of a fluoroquinolone likely provides similar benefits to a prolonged course while decreasing side effects, lowering the risk of antibiotic resistance, and reducing costs to the healthcare system by reducing the number of unnecessary hospital admissions for IV antibiotics. Though a recent prospective trial found no difference between IV versus PO antibiotic prophylaxis for post-operative endophthalmitis in OGI, ${ }^{28}$ IV antibiotics make sense for a one-time dose as patients with OGI may have difficulty tolerating oral medications in the peri-operative period.

While the use of systemic antibiotic prophylaxis is common practice for OGI, the use of intravitreal antibiotic prophylaxis and timing of IOFB removal are related questions that are under debate. Numerous studies have found that the presence of an IOFB alone, regardless of time of removal is an independent risk factor for the development of endophthalmitis. ${ }^{2,3,5,6,15}$ Our study did not find the presence of IOFB to be a predictor for the development of endophthalmitis in multivariable analysis, however, this is likely because our institution utilizes intravitreal antibiotic prophylaxis in cases of IOFB where IOFB removal is delayed. In support of this practice, a multicenter randomized control trial found a benefit of prophylactic intravitreal gentamicin and clindamycin in cases of IOFB. ${ }^{11}$ In 1990, Mieler et al demonstrated in a series of 27 patients that over one-fourth of patients had positive culture growth from their removed IOFB; however, none of their patients developed endophthalmitis. ${ }^{29}$ They advocated an approach of early globe closure and early IOFB removal with systemic antibiotics prophylaxis as well as selective use of intravitreal antibiotics in cases of high-risk injury with soil contamination. In our study, the use of intravitreal antibiotics in cases of delayed IOFB removal likely decreased the microbial load associated with IOFBs enough to prevent the development of endophthalmitis until IOFB removal could occur. Essex et al demonstrated in their 
large series of ocular trauma patients that delayed IOFB removal did not increase the risk of endophthalmitis. ${ }^{6}$ More recently, a study of 79 eyes of soldiers injured in Iraq with IOFB demonstrated that delayed IOFB removal with only systemic and topical antibiotic prophylaxis did not result in an increased rate of endophthalmitis. ${ }^{30}$ In this study, the median time to removal was 21 days and there were no cases of endophthalmitis, though these IOFBs may have been heat sterilized during explosions as a large number were from improvised explosive devices. While removal of IOFBs at time of OGI repair is often the preferred timing, there are many circumstances where this is not possible. Given the results from these studies and our own, we propose that delayed IOFB removal within a few days of globe closure (the median time to removal was two days in our study) with intravitreal antibiotic injection at the time of globe closure may be an acceptable practice in the management of OGI involving IOFBs, especially if it would be unsafe to remove the IOFB primarily at the time of globe closure. In addition to the use of intravitreal antibiotics in cases of IOFBs, it may be reasonable to utilize intravitreal antibiotics in cases of delayed presentation even without IOFB, as our study found delayed presentation to be a risk factor for the development of endophthalmitis.

The microbiology of post-traumatic endophthalmitis has remained relatively stable over the last few decades with the most commonly isolated organisms being Grampositive Bacillus, Streptococcal, and Staphylococcal

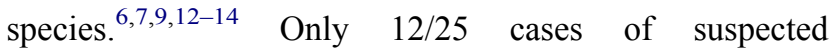
endophthalmitis had positive culture results with the most common organisms being Gram-positive including Bacillus, Staphylococcus, Clostridium, Enterococcus, Streptococcus species, and undifferentiated Gram-positive rods in 10 of these cases. It is interesting to note that of the eyes with endophthalmitis requiring enucleation due to low-likelihood of salvaging the eye, four of five $(80.0 \%)$ had Gram-positive rods. This supports the commonly held belief that Gram-positive rods, including Bacillus species, cause a particularly fulminant form of endophthalmitis. Given the speciation that we encountered in this study, vancomycin and ceftazidime together are a potent combination of intravitreal antibiotics to treat suspected cases of endophthalmitis. All five isolates with sensitivity data were sensitive to vancomycin. Indeed, a review of 665 isolates from cases of endophthalmitis found that $99.7 \%$ of Gram-positive isolates were susceptible to vancomycin and $93.6 \%$ of Gram-negative isolates were susceptible to ceftazidime. $^{31}$

Limitations of this study include those inherent to any retrospective study, specifically, observer bias and at times incomplete records for some patients. The rate of endophthalmitis may be higher in our cohort of patients than those treated at other centers given that we are a tertiary referral center and delays are inevitable as patients first present elsewhere prior to transferring to our center. Our results may not be generalizable, however, as they are based on referrals which yielded a diverse cohort of patients who presented with a variety of injuries, including those from urban or remote rural areas. Another source of potential bias is that a large number of patients diagnosed with OGI during the study period, 407/993, did not meet the inclusion criteria, mostly due to a lack of 30 days of follow-up. All charts of these patients were reviewed to see if any developed endophthalmitis and none did as far as our records show. Given our large catchment area, many of these patients choose to return to their local ophthalmologists for follow-up after their post-operative day one appointment. We suspect that if these patients were to have developed endophthalmitis they would have been referred back to our center for further treatment, but, of course, this is not certain.

The rate of endophthalmitis following OGI has declined over the past 30 years as prompt diagnosis and closure of the globe has become standard of care across the world. ${ }^{2-11}$ Our rate of endophthalmitis, 4.3\%, compares favorably to reports from many large academic centers. $^{2-11}$ Over half of our cases of endophthalmitis were diagnosed at presentation, giving a rate of endophthalmitis developing post-operatively of $2.2 \%$. Given the poor visual outcomes in OGI complicated by endophthalmitis, identification of those eyes most at risk is critical so that they can be closely watched and perhaps prophylactically treated. We found that delayed globe closure and zone I injury were risk factors for the development of endophthalmitis. We did not find that delayed IOFB removal increased the risk of endophthalmitis. Therefore, it may be prudent to utilize prophylactic intravitreal antibiotics in cases of IOFB or delayed presentation. Subconjunctival antibiotics were found to be protective, and this practice is already routinely followed at most institutions during globe closure. We believe that a standardized protocol that utilizes a one-time dose of IV fluoroquinolone antibiotics, globe closure within 24 hours whenever possible, and prophylactic intravitreal 
antibiotics in cases involving delayed IOFB removal provides effective prophylaxis against endophthalmitis in OGI.

\section{Funding}

Heed Ophthalmic Foundation to PYZ.

\section{Disclosure}

The authors report no conflicts of interest in this work.

\section{References}

1. Mir TA, Canner JK, Zafar S, Srikumaran D, Friedman DS, Woreta FA. Characteristics of open globe injuries in the United States from 2006 to 2014. JAMA Ophthalmol. 2020;138 (3):268-275. doi:10.1001/jamaophthalmol.2019.5823

2. Andreoli CM, Andreoli MT, Kloek CE, Ahuero AE, Vavvas D, Durand ML. Low rate of endophthalmitis in a large series of open globe injuries. Am J Ophthalmol. 2009;147(4):601-608.e2. doi:10.1016/j.ajo.2008.10.023

3. Banker TP, McClellan AJ, Wilson BD, et al. Culture-positive endophthalmitis after open globe injuries with and without retained intraocular foreign bodies. Ophthalmic Surg Lasers Imaging Retina. 2017;48(8):632-637. doi:10.3928/23258160-20170802-05

4. Verbraeken H, Rysselaere M. Post-traumatic endophthalmitis. Eur J Ophthalmol. 1994;4(1):1-5. doi:10.1177/112067219400400101

5. Faghihi H, Hajizadeh F, Esfahani MR, et al. Posttraumatic endophthalmitis: report no. 2. Retina. 2012;32(1):146-151. doi:10.1097/IAE.0b013e3182180087

6. Essex RW, Yi Q, Charles PGP, Allen PJ. Post-traumatic endophthalmitis. Ophthalmology. 2004;111(11):2015-2022. doi:10. 1016/j.ophtha.2003.09.041

7. Thompson JT, Parver LM, Enger CL, Mieler WF, Liggett PE. Infectious endophthalmitis after penetrating injuries with retained intraocular foreign bodies. National Eye Trauma System. Ophthalmology. 1993;100(10):1468-1474. doi:10.1016/s01616420(93)31454-5

8. Zhang Y, Zhang MN, Jiang CH, Yao Y, Zhang K. Endophthalmitis following open globe injury. Br J Ophthalmol. 2010;94(1):111-114. doi:10.1136/bjo.2009.164913

9. Sabaci G, Bayer A, Mutlu FM, Karagül S, Yildirim E. Endophthalmitis after deadly-weapon-related open-globe injuries: risk factors, value of prophylactic antibiotics, and visual outcomes. Am J Ophthalmol. 2002;133(1):62-69. doi:10.1016/s0002-9394(01)01320-4

10. Thompson WS, Rubsamen PE, Flynn HW, Schiffman J, Cousins SW. Endophthalmitis after penetrating trauma. Risk factors and visual acuity outcomes. Ophthalmology. 1995;102(11):1696-1701. doi:10. 1016/s0161-6420(95)30807-x

11. Soheilian M, Rafati N, Mohebbi M-R, et al. Prophylaxis of acute posttraumatic bacterial endophthalmitis: a multicenter, randomized clinical trial of intraocular antibiotic injection, report 2. Arch Ophthalmol. 2007;125(4):460-465. doi:10.1001/archopht.125.4.460

12. Jindal A, Pathengay A, Mithal K, et al. Endophthalmitis after open globe injuries: changes in microbiological spectrum and isolate susceptibility patterns over 14 years. J Ophthalmic Inflamm Infect. 2014;4(1):5. doi:10.1186/1869-5760-4-5

13. Long C, Liu B, Xu C, Jing Y, Yuan Z, Lin X. Causative organisms of post-traumatic endophthalmitis: a 20 -year retrospective study. $B M C$ Ophthalmol. 2014;14:34. doi:10.1186/1471-2415-14-34

14. Boldt HC, Pulido JS, Blodi CF, Folk JC, Weingeist TA. Rural endophthalmitis. Ophthalmology. 1989;96(12):1722-1726. doi:10.10 16/s0161-6420(89)32658-3
15. Li X, Zarbin MA, Langer PD, Bhagat N. Posttraumatic endophthalmitis: an 18-year case series. Retina. 2018;38(1):60-71. doi:10.1097/ IAE. 0000000000001511

16. Holladay JT. Visual acuity measurements. $J$ Cataract Refract Surg. 2004;30(2):287-290. doi:10.1016/j.jcrs.2004.01.014

17. Pieramici DJ, Sternberg P, Aaberg TM, et al. A system for classifying mechanical injuries of the eye (globe). The Ocular Trauma Classification Group. Am J Ophthalmol. 1997;123(6):820-831. doi:10.1016/s0002-9394(14)71132-8

18. Meredith TA, Aguilar HE, Shaarawy A, Kincaid M, Dick J, Niesman MR. Vancomycin levels in the vitreous cavity after intravenous administration. Am $J$ Ophthalmol. 1995;119(6):774-778. doi:10.1016/s0002-9394(14)72784-9

19. Aguilar HE, Meredith TA, Shaarawy A, Kincaid M, Dick J. Vitreous cavity penetration of ceftazidime after intravenous administration. Retina. 1995;15(2):154-159. doi:10.1097/00006982-199515020-00 012

20. Hariprasad SM, Shah GK, Mieler WF, et al. Vitreous and aqueous penetration of orally administered moxifloxacin in humans. Arch Ophthalmol. 2006;124(2):178-182. doi:10.1001/archopht.124.2.178

21. El Baba FZ, Trousdale MD, Gauderman WJ, Wagner DG, Liggett PE. Intravitreal penetration of oral ciprofloxacin in humans. Ophthalmology. 1992;99(4):483-486. doi:10.1016/s0161-6420(92) 31943-8

22. Herbert EN, Pearce IA, McGalliard J, Wong D, Groenewald C. Vitreous penetration of levofloxacin in the uninflamed phakic human eye. $\mathrm{Br} J$ Ophthalmol. 2002;86(4):387-389. doi:10.1136/ bjo.86.4.387

23. Elliott JP, Freeman RK, Dorchester W. Short versus long course of prophylactic antibiotics in cesarean section. Am J Obstet Gynecol. 1982;143(7):740-744. doi:10.1016/0002-9378(82)90002-3

24. Yang L, Gao L, Chen Y, et al. Prophylactic antibiotics in prostate biopsy: a meta-analysis based on randomized controlled trials. Surg Infect (Larchmt). 2015;16(6):733-747. doi:10.1089/sur.2015.040

25. Mathur P, Trikha V, Farooque K, et al. Implementation of a short course of prophylactic antibiotic treatment for prevention of postoperative infections in clean orthopaedic surgeries. Indian J Med Res. 2013;137(1):111-116.

26. Khariwala SS, Le B, Pierce BHG, Isaksson Vogel R, Chipman JG. Antibiotic use after free tissue reconstruction of head and neck defects: short course vs. long course. Surg Infect (Larchmt). 2015;17(1):100-105. doi:10.1089/sur.2015.131

27. Cornwell EE, Dougherty WR, Berne TV, et al. Duration of antibiotic prophylaxis in high-risk patients with penetrating abdominal trauma: a prospective randomized trial. J Gastrointest Surg. 1999;3 (6):648-653. doi:10.1016/S1091-255X(99)80088-2

28. Tabatabaei SA, Soleimani M, Behrooz MJ, Sheibani K. Systemic oral antibiotics as a prophylactic measure to prevent endophthalmitis in patients with open globe injuries in comparison with intravenous antibiotics. Retina. 2016;36(2):360-365. doi:10.1097/IAE.000000 0000000727

29. Mieler WF, Ellis MK, Williams DF, Han DP. Retained intraocular foreign bodies and endophthalmitis. Ophthalmology. 1990;97 (11):1532-1538. doi:10.1016/s0161-6420(90)32381-3

30. Colyer MH, Weber ED, Weichel ED, et al. Delayed intraocular foreign body removal without endophthalmitis during Operations Iraqi Freedom and Enduring Freedom. Ophthalmology. 2007;114 (8):1439-1447. doi:10.1016/j.ophtha.2006.10.052

31. Kodati S, Eller AW, Kowalski RP. The susceptibility of bacterial endophthalmitis isolates to vancomycin, ceftazidime, and amikacin: a 23 year-review. Ophthalmol Retina. 2017;1(3):206-209. doi:10. 1016/j.oret.2016.11.010 


\section{Publish your work in this journal}

Clinical Ophthalmology is an international, peer-reviewed journal covering all subspecialties within ophthalmology. Key topics include: Optometry; Visual science; Pharmacology and drug therapy in eye diseases; Basic Sciences; Primary and Secondary eye care; Patient Safety and Quality of Care Improvements. This journal is indexed on PubMed
Central and CAS, and is the official journal of The Society of Clinical Ophthalmology (SCO). The manuscript management system is completely online and includes a very quick and fair peer-review system, which is all easy to use. Visit http://www.dovepress.com/ testimonials.php to read real quotes from published authors. 